\title{
Soliton Turbulence as a Thermodynamic Limit of Stochastic Soliton Lattices
}

\author{
Gennady A. El \\ Institute of Terrestrial Magnetism, Ionosphere and Radio Wave Propagation, Russian \\ Academy of Sciences, Troitsk, Moscow Region, e-mail: el@izmiran.rssi.ru \\ Alexander L. Krylov \\ O.Yu. Shmidt Institute of Earth Physics, Russian Academy of Sciences, Moscow \\ Stanislav A. Molchanov \\ North Carolina University at Charlotte \\ Stephanos Venakides \\ Duke University, Durham, NC
}

\begin{abstract}
We use recently introduced notion of stochastic soliton lattice for quantitative description of soliton turbulence. We consider the stochastic soliton lattice on a special band-gap scaling of the spectral surface of genus $N$ so that the integrated density of states remains finite as $N \rightarrow \infty$ (thermodynamic type limit). We prove existence of the limiting stationary ergodic process and associate it with the soliton turbulence. The phase space of the soliton turbulence is a one-dimensional space with the random Poisson measure. The zero density limit of the soliton turbulence coincides with the Frish - Lloyd potential of the quantum theory of disordered systems.
\end{abstract}

\section{Introduction}

The idea of introducing the statistical description into the soliton theory has its origin in the work of Zakharov [1] on kinetic equation for the rarefied soliton gas. In this work, a new object, an infinite sequence of KdV solitons on the $x$-axis was considered. An accurate limit for the $N$ - soliton solution as $N \rightarrow \infty$ is , however, very nontrivial and has been investigated so far only for the case of a very special phase distribution [2], [3] which yields the reflectionless potential decaying at one of infinities $x \rightarrow \pm \infty$. This kind of infinite - soliton solutions does not provide directly the spatial uniformity which is necessary for description of the soliton gas in thermodynamically equilibrium state.

Another view on the problem was proposed by Lax [4] who noticed that 'the weak limits of oscillatory sequences of dispersive compressible flows show a remarkable resemblance to ensemble averages of classical turbulence theory'. To constitute such a deterministic analogue of turbulence one has to construct the correspondent ensemble of flows and provide it with the physically plausible measure which form a stochastic process. It is clear from the very 
beginning that for description of the 'soliton turbulence' we are interested in the stationary ergodic processes.

The consistent way of application of stochastic description to the soliton theory was proposed in [5], [6] where the notion of stochastic soliton lattice (SSL) was introduced. The basic idea of such a description lies in the fact that the finite-gap solutions of completely integrable equations are almost periodic functions and posess, therefore, their natural stochastic structure [7] determined by the compact shift group with the uniform (Haar) measure.

In this work, we investigate $N$-gap stochastic soliton lattices $q(x)=\nu_{N}(x)$ of the $\mathrm{KdV}$ equation on a special band-gap scaling of the spectral Riemann surface of the complex parameter $E$ when the width of gaps is $\sim O(1 / N)$ while the bands are exponentially narrow $\sim \exp (-N), N \gg 1$. Along with this, the small parameter $1 / N$ in our consideration does not appear explicitely in the Schrödinger equation $\left(-d_{x x}^{2}+q(x)\right) \phi=E \phi$.

The basic results of the present work are:

- the process $\zeta(x)=\lim _{N \rightarrow \infty} \nu_{N}(x)$ exists. It is an ergodic stationary random process each realization of which satisfies the KdV equation. The phase space of $\zeta(x)$ is a one-dimensional space with random Poisson measure.

- the considered limit is of a thermodynamic type (the integrated density of states remains finite as $N \rightarrow \infty$ for the chosen scaling of the band-gap stucture).

- as $\lim _{N \rightarrow \infty} \frac{\text { band }}{\text { gap }} \rightarrow 0$ the thermodynamic limit of stochastic soliton lattices $\zeta(x)$ can be interpreted as a soliton turbulence.

- we calculate the rotation number and some ensemble averages for the soliton turbulence.

- we show that the zero-density limit of the soliton turbulence yields the Frish - Lloyd potential of the quantum theory of disordered systems [9], [10].

\section{Basic Preliminaries}

The soliton lattice (SL) ( $N$-gap potential of the Schrödinger equation) is given by the known Its - Matveev formula (see for instance [11])

$$
\begin{gathered}
u_{N}(x ; \mathbf{r})=C(\mathbf{r})-2 \partial_{x x}^{2} \log \Theta[\mathbf{z}(\mathbf{x}) \mid B(\mathbf{r})], \\
x \in \mathbf{R}, \quad \mathbf{r}=\left(r_{1}, \ldots, r_{2 N+1}\right), \quad r_{1}<r_{2}<\ldots<r_{2 N}<r_{2 N+1} .
\end{gathered}
$$

Here

$$
\begin{gathered}
\Theta[\mathbf{z} \mid B(\mathbf{r})]=\sum_{\mathbf{m}} \exp \{\pi i[2(\mathbf{m}, \mathbf{z})+(\mathbf{m}, B \mathbf{m})]\} \\
\mathbf{m}=\left(m_{1}, \ldots, m_{N}\right) \in \mathbf{Z}^{N}, \quad \mathbf{z} \in \mathbf{C}^{N}
\end{gathered}
$$


is the Jacobi theta-function of the hyperelliptic Riemann surface of genus $N$

$$
\mu^{2}=\prod_{j=1}^{2 N+1}\left(E-r_{j}\right) \equiv R_{2 N+1}(E, \mathbf{r})
$$

with cuts along the bands.

The Riemann matrix $B(\mathbf{r})$ and the constant $C(\mathbf{r})$ are expressed in terms of the basis holomorphic differentials $\psi_{j}$ :

$$
B_{i j}=\oint_{\beta_{j}} \psi_{i}, \quad C(\mathbf{r})=\sum_{j=1}^{N} r_{j}-2 \sum_{j=1}^{N} \oint_{\alpha_{j}} E \psi_{j},
$$

Here

$$
\psi_{j}=\sum_{k=0}^{N-1} a_{j k} \frac{E^{k}}{\sqrt{R_{2 N+1}(\mathbf{r}, E)}} d E,
$$

and dependence $a_{j k}(\mathbf{r})$ is given by the normalization

$$
\oint_{\alpha_{k}} \psi_{j}=\delta_{j k}
$$

where the $\alpha$-cycles surround the bands clockwise, and the $\beta$-cycles are canonically conjugated to $\alpha$ 's such that the contour $\beta_{j}$ starts from the $j$-th cut, then goes to $+\infty$ and returns on the lower cut.

The imaginary phases $\mathbf{z}(x)$ are given by the formula

$$
\mathbf{z}=-2 i \mathbf{a}_{N-1} x+\mathbf{d},
$$

where $\mathbf{d}$ is the initial imaginary phase vector.

Using the substitution

$$
\mathbf{z}=\frac{1}{2 \pi} B \mathbf{y}
$$

we rewrite (1) in the form with real phases $\mathbf{y}$ [12

$$
\begin{gathered}
u_{N}(x \mid \mathbf{r})=u_{N}\left(y_{1}(x), \ldots, y_{N}(x) \mid \mathbf{r}\right), \\
y_{j}(x)=k_{j} x+f_{j}, \quad f_{j}(\bmod 2 \pi), \\
\mathbf{k}=-4 \pi i B^{-1} \mathbf{a}_{N-1} .
\end{gathered}
$$

Here $\mathbf{k}=\mathbf{k}(\mathbf{r})=\left(k_{1}, \ldots, k_{N}\right)$ is the wave number vector, and $\mathbf{f}=\left(f_{1}, \ldots, f_{N}\right)$ is the initial (angle) phase vector, $-\pi<f_{j} \leq \pi$. Note that in (9)

$$
u_{N}\left(y_{1}, \ldots, y_{j}+2 \pi, \ldots, y_{N} \mid \mathbf{r}\right)=u_{N}\left(y_{1}, \ldots, y_{j}, \ldots, y_{N} \mid \mathbf{r}\right)
$$


that is $u_{N}(x \mid \mathbf{r})$ is $N$-quasiperiodic in $x$ 13], 14 (hereafter we consider only incommensurate $\left.k_{j}, j=1, \ldots, N\right)$. As for any almost periodic function, we have for $u_{N}(x \mid \mathbf{r})$ the Fourier representation

$$
u_{N}(x \mid \mathbf{r})=\sum_{j} c_{j} e^{i\left(l_{j} x+h_{j}\right)},
$$

where $c_{j}, l_{j}, h_{j}$ are real, $-\pi<h_{j} \leq \pi$. $\left(l_{j} \in \mathbf{M}\right.$, where $\mathbf{M}$ is a frequency-module [8] and $h_{j}$ are the integer linear combinations of $\left.f_{j}(\bmod 2 \pi)\right)$.

The KdV evolution of (9) is isospectral and is described by the linear motion of the phases on the Jacobian:

$$
\begin{gathered}
u_{N}(x, t \mid \mathbf{r})=\left(y_{1}(x, t), \ldots, y_{N}(x, t) \mid \mathbf{r}\right), \\
y_{j}(x, t)=k_{j} x+\omega_{j} t+f_{j},
\end{gathered}
$$

where the frequency vector

$$
\omega=\omega(\mathbf{r})=\left(\omega_{1}, \ldots, \omega_{N}\right)=-8 \pi i B^{-1}\left(\mathbf{a}_{N-1} \sum_{j=1}^{2 N+1} r_{j}+2 \mathbf{a}_{N-2}\right) .
$$

\section{Stochastic Soliton Lattices}

It is well known that any almost periodic function generates the stochastic stationary process (see [8], [7]).

Definition 1. [5], [6] The stochastic process generated by SL $u_{N}(x \mid \mathbf{r})$ we call Stochastic Soliton Lattice (SSL) and denote as $\nu_{N}(x \mid \mathbf{r})$.

The general construction of $\nu_{N}(x \mid \mathbf{r})$ admits a very simple and clear description. The realization set $\Omega$ of it consists of functions (9), (10) where $\mathbf{f} \in \operatorname{Tor}^{N} ; \operatorname{Tor}^{N}$ is $N$-dimensional torus $(-\pi, \pi]^{N}$. The probability measure $d \mu$ is the uniform (Lebesque) measure on the torus. It corresponds to the description of $\nu_{N}(x \mid \mathbf{r})$ following from(9),(10):

$$
\nu_{N}(x \mid \mathbf{r})=u_{N}\left(\ldots k_{j} x+\phi_{j} \ldots \mid \mathbf{r}\right)
$$

where $\phi_{1}, \ldots, \phi_{N}$ are independent random values uniformly distributed on $(-\pi, \pi]$, that is $\phi=\left(\phi_{1}, \ldots, \phi_{N}\right)$ is uniformly distributed on $\operatorname{Tor}^{N}$. As $k_{j}$ are incommensurate then $\nu_{N}(x \mid \mathbf{r})$ is an ergodic process [16].

As $\nu_{N}(x \mid \mathbf{r})$ is the stationary process then it has the Stone - Kolmogorov spectral decomposition [15]; due to the ergodicity this decomposition has the form (cf. (13))

$$
\nu_{N}(x \mid \mathbf{r})=\sum_{j} c_{j} e^{i\left(l_{j} x+\theta_{j}\right)},
$$

where $\theta_{j}$ are uniformly distributed on $(-\pi, \pi]$ noncorrelated random values [17. 
The well known formula (Bochner - Khintchin) [15] gives us the covariance function $K(h)$ of the stationary process $\nu_{N}(x \mid \mathbf{r})$ :

$$
K(h) \equiv\left\langle\hat{\nu}_{N}(x \mid \mathbf{r}) \cdot \hat{\nu}_{N}(x+h \mid \mathbf{r})\right\rangle=\sum_{j}\left|c_{j}\right|^{2} e^{i l_{j} h} .
$$

Here $\hat{\xi}(x) \equiv \xi(x)-\langle\xi\rangle$ is the centered process.

Theorem. Consider the KdV equation $u_{t}-6 u u_{x}+u_{x x x}=0$ as the equation describing the evolution in the phase space of stationary processes. Let the initial data has the form of the SSL:

$$
u(x, 0)=\nu_{N}(x \mid \mathbf{r})
$$

Then the solution of the $\mathrm{KdV}$ is

$$
u(x, t)=\nu_{N}(x \mid \mathbf{r})=u(x, 0)
$$

that is $u(x, 0)=\nu_{N}(x \mid \mathbf{r})$ is a stationary point.

Proof . The evolution of realizations (9), (10) is described by (14),(15). For any moment $t$ one can introduce the new 'initial phase' $f_{j}^{*}=\omega_{j} t+f_{j}$ which is also uniformly distributed on $\operatorname{Tor}^{N}$. Therefore, the KdV evolution changes neither realization set $\Omega$ nor the probability measure. Q.E.D.

Now we present some expressions for the ensemble averages which will be needed in the future. If $Q(f)$ is an arbitrary smooth function then the mean value $Q(\xi)$ of the stochastic process $\xi$ is

$$
\langle Q(\xi)\rangle=\int_{\Omega} d \mu Q(f), \quad f \in \Omega .
$$

(We recall that $\xi(x) \equiv\{\Omega=\{f(x)\}, B, \mu\}$, and $B$ is some $\sigma$-algebra of measurable Borel sets of $\Omega$ ).

For $\nu_{N}(x \mid \mathbf{r})$ we have 12

$$
\left\langle Q\left(\nu_{N}(x \mid \mathbf{r})\right\rangle=\frac{1}{(2 \pi)^{N}} \int_{-\pi}^{\pi} \ldots \int_{-\pi}^{\pi} d \phi_{1} \ldots d \phi_{N} Q\left(\nu_{N}(x \mid \mathbf{r})\right)=\lim _{L \rightarrow \infty} \frac{1}{L} \int_{0}^{L} Q\left(\nu_{N}(x \mid \mathbf{r})\right),\right.
$$

which constitutes ergodicity of SSL. Direct calculation using (1),(2) gives surprisingly simple formulas for two first moments of the value $\nu_{N}(x \mid \mathbf{r})$ Namely,

$$
\left\langle\nu_{N}(x \mid \mathbf{r})\right\rangle=C(\mathbf{r})-\frac{1}{\pi i}(\mathbf{k}, B \mathbf{k}), \quad\left\langle\nu_{N}^{2}(x \mid \mathbf{r})\right\rangle=-\frac{1}{3 \pi i}(\omega, B \mathbf{k}),
$$

and the vectors $\mathbf{k}(\mathbf{r}), \omega(\mathbf{r})$ are given by (11), (16). It should be noted that expressions (24) are obtained for the particular choice of the canonical basis of cycles (see (6)). However, namely this normalizatiton is preferrable for our consideration. 


\section{Rotation Number in Stochastic Soliton Lattices}

Consider the Schrödinger equation with an almost periodic potential $q(x)$

$$
\left(-\partial_{x x}^{2}+q(x)\right) \phi=E \phi, \quad x \in \mathbf{R} .
$$

The potential $q(x)$ has an important characteristics, the rotation number, which is defined for real $E$ as $[\mathrm{JM}]$

$$
\alpha(E)=\lim _{x \rightarrow \infty} \frac{1}{x} \arg \left(\phi^{\prime}(x, E)+i \phi(x, E)\right) .
$$

We will use also the integrated density of states $\mathcal{N}(E)$ which is connected with the rotation number by a simple relation (here we improve some inaccuracies in [5], [6]):

$$
\mathcal{N}(E)=\frac{1}{\pi} \alpha(E) .
$$

If $q(x)$ is N-gap potential , $q(x)=u_{N}(x \mid \mathbf{r})$, then

$$
\alpha_{N}(E)=\operatorname{Re} \int_{-\infty}^{E} d p\left(E^{\prime}\right)
$$

where $d p(E)$ is the quasimomentum differential [12], [11]:

$$
d p(E)=\frac{E^{N}+b_{N-1} E^{N-1}+\ldots+b_{0}}{\sqrt{R_{2 N+1}(E ; \mathbf{r})}}, \quad \oint_{\beta_{j}} d p(E)=0, \quad j=1, \ldots, N .
$$

The integrals of $d p$ over the $\alpha$ - cycles are known [12], [11] to give the components of the wave number vector (11)

$$
\oint_{\alpha_{j}} d p(E)=k_{j} .
$$

Then,

$$
\begin{aligned}
& \frac{1}{2} \sum_{j=1}^{M(E)} k_{j} \quad \text { if } E \in \operatorname{gap}_{M} \\
& \alpha_{N}(E)= \\
& \frac{1}{2} \sum_{j=1}^{M(E)} k_{j}+\int_{r_{2 M-1}}^{E} d p\left(E^{\prime}\right) \quad \text { if } E \in \operatorname{band}_{M},
\end{aligned}
$$

where $M(E)$ is the number of the band nearest to $E$ from the left $M(E) \leq N, M(0)=$ $N, M(-1)=0$.

We note that the formula (31) gives an effectivization for the case of finite-gap potentials of the statement in [8] that the values of the function $2 \alpha(E)$ if $E \in\{$ gap $\}$ belong to the frequency module of the almost periodic potential. 
As the SL (9), (10) is the quasiperiodic function both in $x$ and $t$ one can also formally introduce the temporal rotation number for the SSL by

$$
\alpha_{N}^{t}(E)=\operatorname{Re} \int_{-\infty}^{E} d q\left(E^{\prime}\right)
$$

where $d q(E)$ is the quasienergy differential [12], [1] $\left(d q(E)=\left(E^{N+1}+c_{N} E^{N}+\ldots+\right.\right.$ $\left.c_{0}\right) d E / \sqrt{R_{2 N+1}(E ; \mathbf{r})}, \oint_{\beta_{j}} d q(E)=0, \quad \oint_{\alpha_{j}} d q(E)=\omega_{j}$, where $\omega_{j}$ are the frequences (16)).

Since the value of $\alpha_{N}(E)$ is the same for any realization from the set $\Omega$ from Def.1 then it is the spectral characteristics of the whole SSL $\nu_{N}(x \mid \mathbf{r})$ (deterministic characteristics of the stochastic process). Due to the Theorem 1 the rotation number does not change under the KdV evolution.

We introduce also the full integrated density of states in the SSL $\lambda$ which is a number:

$$
\lambda \equiv \mathcal{N}_{N}(\infty)=\frac{1}{2 \pi} \sum_{j=1}^{N} k_{j}
$$

One can see that the full integrated density of states has in the SSL the natural meaning of the mean number of waves per unit length.

\section{Thermodynamic Limit of Stochastic Soliton Lattices}

Let the nontrivial (band-gap) part of the spectrum lies in the interval $(-1,0)$ of the real $E$-axis: $\alpha_{N}(-1)=0, \alpha_{N}(0)=\pi \lambda$. For $N \gg 1$ we consider the following scaling of the band-gap structure

$$
\operatorname{gaps}(E) \sim \frac{1}{N} \quad \operatorname{bands}(E) \sim \exp (-N) \quad N \gg 1, \quad E \in(-1,0) .
$$

Using (11) one can show that the scaling (34) implies the following behavior for the wave numbers: $k_{j} \sim 1 / N$, which provides finiteness of the rotation number $\alpha_{N}(E)$ (and of the integrated density of states $\left.\mathcal{N}_{N}(E)\right)$ as $N \rightarrow \infty$ :

$$
\alpha(E) \equiv \lim _{N \rightarrow \infty} \alpha_{N}(E)=\lim _{N \rightarrow \infty} \frac{1}{2} \sum_{j=1}^{M(E)} k_{j}<\infty, \quad M \leq N
$$

Due to this property we call the scaling (34) and the correspondent limit as $N \rightarrow \infty$ the thermodynamic ones.

It should be noted that the thermodynamic scaling (34) appears when one considers the quasiclassical asymptotics of the spectrum for periodic potentials [18], [19], [20]. In contrast to indicated works, however, the small parameter $1 / N$ in our consideration is contained only in the band-gap structure of the spectrum and does not appear explicitely in the Schrödinger equation (25). As a result, we will show that the limit for the finite-gap potentials on the 
thermodynamic scaling exists as $N \rightarrow \infty$ in a strong sense. We emphasize the difference of this point from the known Lax - Levermore - Venakides (LLV) approach (see [21], [19], [20]) to the limiting passage $\lim _{N \rightarrow \infty} u_{N}$ where due to rescaling $x \rightarrow N x$ the above limit exists only in a weak sense. The LLV consideration provides information about the slow modulations (macrostructure) appearing in the $N$-soliton (or $N$-gap) KdV solution under the time evolution. We, on the contrary, are interested in the limiting microstructure of nonmodulated $N$-phase wave as $N \rightarrow \infty$. Futhermore, we are going to show that the thermodynamic limit exists for the whole stochastic process (SSL) which implies not only compactness of the realization set but also convergence of the probability measure.

Now we describe the thermodynamic scaling more in detail. Following [20] we introduce the lattice of points

$$
1 \approx \eta_{1}>\eta_{2}>\ldots>\eta_{N} \approx 0
$$

where

$$
-\eta_{j}^{2}=\frac{1}{2}\left(r_{2 j-1}+r_{2 j}\right)
$$

are centers of bands.

We define two continuous functions on the lattice:

1. The normalized density of bands: $\varphi(\eta) d \eta \approx \frac{\text { number of bands in }(E, E+d E)}{N}$

$$
\varphi\left(\eta_{j}\right)=\frac{1}{N\left(\eta_{j}-\eta_{j+1}\right)}+O\left(\frac{1}{N}\right), \quad \int_{0}^{1} \varphi(\eta) d \eta=1, \quad \eta^{2}=-E \in(0,1) .
$$

2. The normalized logarithmic band width

$$
\gamma\left(\eta_{j}\right)=-\frac{1}{N} \log \left(r_{2 j}-r_{2 j-1}\right)+O\left(\frac{1}{N}\right) .
$$

Now one can easily represent the relationship (35) for the thermodynamic limit of the rotation number in a continuum form:

$$
2 d \alpha\left(-\eta^{2}\right)=\varphi(\eta) k(\eta) d \eta
$$

where $k(\eta)$ is the continu of the wave number vector (11).

For the temporal rotation number $\alpha_{N}^{t}(E)$ (32) in the thermodynamic limit we have the equation which is analogous to (39):

$$
2 d \alpha^{t}\left(-\eta^{2}\right)=\varphi(\eta) \omega(\eta) d \eta
$$

where $\omega(\eta)$ is the continuum limit for the frequency vector (16).

To find a continuum limit for the wave number and the frequency vectors given by (11) and (16) one has to know the continuum limits of the period matrix $B$ (困) and of the vectors $\mathbf{a}_{N-1}$ and $\mathbf{a}_{N-2}$ from (5). It is clear that all quantities given on the Riemann surface can be computed now in terms of two functions (densities) $\varphi(\eta)$ and $\gamma(\eta)$ completely describing the band - gap structure. 
In particular, the period matrix (田) and the vectors $\mathbf{a}_{N-1}$ and $\left(\mathbf{a}_{N-1} \sum_{j=1}^{2 N+1} r_{j}+2 \mathbf{a}_{N-2}\right)$ take the form [20]:

$$
B \rightarrow B(\eta, \mu)=-\frac{i}{\pi}\left(\log \left|\frac{\eta-\mu}{\eta+\mu}\right| \varphi(\mu)+\gamma(\mu) \delta(\eta-\mu)\right),
$$

where $\delta(x)$ is the Dirac delta-function;

$$
\mathbf{a}_{N-1} \rightarrow-\frac{\eta}{2 \pi}, \quad \mathbf{a}_{N-1} \sum_{j=1}^{2 N+1} r_{j}+2 \mathbf{a}_{N-2} \rightarrow \frac{2 \eta^{3}}{\pi}
$$

Now we represent the general relationship (11) between the wave number vector $\mathbf{k}$ and the vector $\mathbf{a}_{N-1}$ in the form suitable for the limiting transition

$$
\mathbf{k} B=-4 \pi i \mathbf{a}_{N-1}
$$

Then applying (39), (41), (42) to (43) we arrive at the integral equation for the rotation number in the thermodynamic limit :

$$
\frac{1}{\pi} \varphi(\eta) d \eta \int_{0}^{1} \log \left|\frac{\eta-\mu}{\eta+\mu}\right| d \alpha\left(-\mu^{2}\right)+\gamma(\eta) d \alpha\left(-\eta^{2}\right)=-\eta \varphi(\eta) d \eta
$$

Similarly, for the thermodynamic limit of the temporal rotation number one gets with the aid of (40), (41), (42) and (16):

$$
\frac{1}{\pi} \varphi(\eta) d \eta \int_{0}^{1} \log \left|\frac{\eta-\mu}{\eta+\mu}\right| d \alpha^{t}\left(-\mu^{2}\right)+\gamma(\eta) d \alpha^{t}\left(-\eta^{2}\right)=4 \eta^{3} \varphi(\eta) d \eta .
$$

Thus, we have established the existence of the thermodynamic limit for the rotation number in SSL (as a matter of fact, the rotation number, as well as in the finite-gap case, is a deterministic function).

The rotation number is known to be an imaginary part of the Floquet exponent $w(E)$ (for the finite-gap potentials $w(E)=i \int d p(E)$ ) [8]. Then we have with the aid of Herglotz formula:

$$
w(E)=\int_{-\infty}^{\infty} \frac{\alpha(z) d z}{E-z}, \quad \operatorname{Im} E>0
$$

But following Kotani [22], if the Floquet exponent satisfies the conditions

$$
\begin{aligned}
& \operatorname{Rew}(E+i 0)=0 \text { a.e. on }[0,+\infty) \\
& \int_{-\infty}^{0}-\operatorname{Rew}(E+i 0) d \alpha(E)=0
\end{aligned}
$$


then there exists an ergodic stationary random process whose Floquet exponent is $w(E)$. One can see that for SSL conditions (47) are satisfied due to the properties of the quasimomentum (29). Therefore the thermodynamic limit of SSL exists.

Remark. It can be shown that the existence of the limit for the rotation number guarantees the existence of the limit for the Weyl function. Then the compactness of the realization set can be deduced from the results of Marchenko [23].

As band/gap $\rightarrow 0$ in the thermodynamic limit (see (34)), then it is natural to call the thermodynamic limit $\zeta(x)$ of the SSL $\nu_{N}(x)$ a soliton turbulence

$$
\zeta(x)=\lim _{N \rightarrow \infty} \nu_{N}(x) .
$$

We believe that the spectrum of a typical realization of the soliton turbulence is pure point on $(-1,0)$ but exact proof of this conjecture is missed yet.

The ensemble averages (moments) (22) are the important characteristics of the soliton turbulence. To compute three first moments we make use of the expressions (24) obtained for the finite-gap SSL. These expressions admit direct limiting passage as $N \rightarrow \infty$ in the thermodynamic scaling (34).

Using results of [20] it is not hard to show that the constant $C(\mathbf{r})$ (四) in the Its-Matveev formula (1) vanishes in the thermodynamic limit. Then, substituting (39), (40) into (24) we have for the two first moments in soliton turbulence:

$$
\begin{gathered}
\langle\zeta\rangle=-2 \lambda \int_{-1}^{0} d \tilde{\alpha}(E)=-2 \lambda, \\
\left\langle\zeta^{2}\right\rangle=\frac{4 \lambda}{3} \int_{-1}^{0} E d \tilde{\alpha}(E)=-\frac{4 \lambda}{3} \bar{E} .
\end{gathered}
$$

Here

$$
d \tilde{\alpha}(E)=\frac{d \alpha}{\pi \lambda}, \quad \int_{-1}^{0} d \tilde{\alpha}=1, \quad \overline{f(E)}=\int_{-1}^{0} f(E) d \tilde{\alpha}(E) .
$$

Thus, we have expressed the ensemble averages in soliton turbulence through the averages over the spectrum with the measure $d \tilde{\alpha}(E)$. One should also note that the Floquet exponent $w(E)$ (46) provides the averaged Kruskal integrals as the coefficients in the decomposition as $E \rightarrow \infty$.

\section{Poissonic Properties of Soliton Turbulence}

We study the phase space of the soliton turbulence. We recall that in the finite-gap SSL the phase space is the $N$ - dimensional torus with the uniform (Lebesque) measure. For performing the thermodynamic limit it is convenient to introduce the linear phases instead of the angle phases $\phi_{j}$ in the SSL (17) 


$$
l_{j} \equiv \frac{\phi_{j}}{k_{j}}
$$

where $l_{j}(j=1, \ldots, N)$ are independent random values uniformly distributed on $\left(-\frac{\pi}{k_{j}}, \frac{\pi}{k_{j}}\right]$ respectively.

The further consideration is close to standard procedure of the thermodynamic limiting passage in the ergodic theory of ideal gas (see for instance [24, [16]). We now turn to a new space with the aid of factorization of the torus by interchange group $S_{N}$. The sets of $N$ points $l_{j}$ serve now as the points of the obtained space $Q_{N}=\operatorname{Tor}^{N} / S_{N}$. The measurable sets in the factorized space consist of realizations having $0,1, \ldots, N$ linear phases in the interval $\Delta$ of the $x$ axis.

We introduce the random value $\xi_{j}=\chi_{(0,1)}\left(l_{j}\right)$ which is the number of hitting of the particular phase $l_{j}$ into the fixed interval $(0,1) \subset \mathbf{R}$ (we suppose that $\pi / k_{j} \geq 1$ ). The variable $\xi_{j}$ takes two values: 1 and 0 with the probabilities $p_{j}(1)=k_{j} / 2 \pi$ and $p_{j}(0)=q_{j}=$ $1-p_{j}=1-k_{j} / 2 \pi$. The generating function $\varphi_{j}(z)$ for $\xi_{j}$ is

$$
\varphi_{j}(z)=\left(1-p_{j}\right)+z p_{j}
$$

The sum $\xi^{(N)} \equiv \sum_{j=1}^{N} \xi_{j}$ is the number of hitting of all linear phases into $(0,1)$. As $\xi_{j}$ are independent random values, then the generating function for $\xi^{(N)}$ has the form

$$
\varphi^{(N)}(z)=\prod_{j=1}^{N} \varphi_{j}(z)=\prod_{j=1}^{N}\left(1+(z-1) p_{j}\right)=\prod_{j=1}^{N}\left(1+\frac{(z-1) k_{j}}{2 \pi}\right) .
$$

On the thermodynamic scaling (34) $k_{j}=O\left(N^{-1}\right)$. Then taking the logarithm of (53) we obtain

$$
\ln \varphi^{(N)}(z)=(z-1) \frac{1}{2 \pi} \sum_{j=1}^{N} k_{j}+O\left(N^{-1}\right) .
$$

Therefore taking into account (33) one has

$$
\varphi^{(\infty)}(z)=\exp \{(z-1) \lambda\}=\sum_{n=0}^{\infty} z^{n}\left(\frac{e^{-\lambda} \lambda^{n}}{n !}\right) .
$$

Thus, the right-hand part of (54) is the generating function for the Poisson distribution with the parameter $\lambda$ ( which is the full integrated density of states in our case). Therefore, the limiting measure in the configurational space is the random Poisson measure (Poissonic white noise).

We note that the Poissonic white noise can be described as the random collection of the points $\left\{l_{j}\right\}$ (the linear phases in our case) on the $x$ - axis such that the distances $s_{k}$ between them are independent random values distributed exponentially with the density

$$
f(s)=\lambda \exp (-\lambda s)
$$


We also note that the Poisson parameter $\lambda$ according to (49) can be expressed through the first moment in the soliton turbulence:

$$
\lambda=-\frac{\langle\zeta\rangle}{2}
$$

The value $\lambda$ can be then interpreted as a density of the soliton turbulence.

\section{The Frish - Lloyd Potential as a Zero-Density Limit of the Soliton Turbulence}

We consider the soliton turbulence with the small integrated density of states. We introduce a small parameter $\varepsilon \ll 1$ and make a renormalization:

$$
\alpha(E)=\varepsilon \tilde{\alpha}(E), \quad \lambda=\varepsilon \tilde{\lambda}
$$

This implies the following transformations for the functions $\varphi(\eta)$ and $\gamma(\eta)$ (37), (38) characterizing the Riemann surface in the thermodynamic scaling

$$
\varphi(\eta)=\varphi(\eta), \quad \gamma(\eta)=\frac{\tilde{\gamma}(\eta)}{\varepsilon}, \quad \tilde{\gamma}(\eta)=O(1)
$$

We choose $\tilde{\gamma}(\eta)=c \eta$ which corresponds to imposing the periodicity condition : $\forall k_{j}=$ $2 \pi c^{-1}\left(\varepsilon N^{-1}\right)$. Then, it follows from (33), (56) that $\tilde{\lambda}=1 / c$.

We also make rescaling of the spatial variables

$$
x=\frac{\tilde{x}}{\varepsilon}, \quad l_{j}=-\frac{\tilde{l}_{j}}{\varepsilon} .
$$

Consider now the SSL with a small integrated density of states on the thermodynamic scaling (34). We make use of the Its - Matveev formula (11):

$$
\nu_{N}(\tilde{x}) \approx-2 \varepsilon^{2} \partial_{\tilde{x} \tilde{x}}^{2} \log \Theta_{N}\left(\ldots, \frac{-2 i a_{N-1, j}\left(\tilde{x}-\tilde{l}_{j}\right)}{\varepsilon}, \ldots \mid B\right),
$$

where according to (41), (42)

$$
\begin{gathered}
B_{j j} \approx \frac{\tilde{\gamma}\left(\eta_{j}\right)}{\varepsilon}, \quad B_{i j} \approx \log \left|\frac{\eta_{i}-\eta_{j}}{\eta_{i}+\eta_{j}}\right|, \\
a_{N-1, j} \approx-\frac{\eta_{j}}{2 \pi} .
\end{gathered}
$$

An analysis of the explicit expression for the theta-function (2) provided (60), (61) shows that on the set of realizations excluding the set of a small measure one can neglect the 
contribution of the off-diagonal part of the period matrix $B$ into the solution (59) (one suppose the inequality

$$
\sum_{j=1}^{N} \frac{\tilde{\gamma}\left(\eta_{j}\right)}{\varepsilon} n_{j}^{2} \gg \sum_{i \neq j=1}^{N} \log \left|\frac{\eta_{i}-\eta_{j}}{\eta_{i}+\eta_{j}}\right| n_{i} n_{j}, \quad N \gg 1 .
$$

to be satisfied outside of the indicated set of a small measure).

Then asymptotically in $N, \varepsilon,(N \gg 1 \varepsilon \ll 1, N \varepsilon \gg 1)$ the following factorization is valid :

$$
\Theta_{N} \approx \prod_{j=1}^{N} \Theta\left(\eta_{j} \frac{\left(\tilde{x}-\tilde{l}_{j}\right)}{\pi \varepsilon} \mid \tau_{j}\right)
$$

where $\Theta(y)$ is the one-dimensional theta-function $\left(\Theta_{3}\right.$ in standard literature (see for ex. 25)),

$$
\tau_{j}=\frac{K\left(1-m_{j}\right)}{K\left(m_{j}\right)} \approx 0, \quad m_{j} \approx 1-\frac{1}{\eta_{j}} \exp \left(-\frac{c}{\varepsilon} \eta_{j}\right) \approx 1 .
$$

Here $K(m)$ is the complete elliptic integral of the first kind, $m_{j}$ are the ellipticity parameters (do not confuse with the multiintegers in (2), (62) ); $m_{j}=1$ corresponds to the soliton limit.

Consider the rescaled SSL of a small density $\nu_{N}(\tilde{x}) / \varepsilon^{2}$. Then it follows from (59), (63), (64) that

$$
\frac{\nu_{N}(\tilde{x})}{\varepsilon^{2}} \approx-2 \sum_{j=1}^{N} \eta_{j}^{2} \frac{\operatorname{sech}^{2}\left(\eta_{j} \frac{\tilde{x}-\tilde{l}_{j}}{\pi \varepsilon}\right)}{\varepsilon^{2}}
$$

Then passing to a limit

$$
\varepsilon \rightarrow 0, \quad N \rightarrow \infty, \quad \varepsilon N \rightarrow \infty
$$

one obtains

$$
\tilde{\zeta}(\tilde{x})=\lim \frac{\nu_{N}(\tilde{x})}{\varepsilon^{2}}=-4 \sum_{j} a_{j}^{2} \delta\left(\tilde{x}-\tilde{l}_{j}\right),
$$

where, according to the results of Sec. $5, \tilde{l}_{j}$ are random points on the $\tilde{x}$ - axis. The distances $\tilde{s}_{k}$ between these points are independent random values distributed with the density

$$
f(\tilde{s})=\frac{1}{c} \exp \left(-\frac{\tilde{s}}{c}\right)
$$

The amplitudes $a_{j}$ are independent among themselves and independent of $\tilde{l}_{j}$ random values distributed with the density $\varphi(a)$. The distribution (67) is so-called complex Poisson white noise; it is called the Frish - Lloyd potential in the quantum theory of disordered systems [9], [10].

\section{Aknowledgements}

We are grateful to M. Freidlin, P.Miller and S.Novikov for useful discussions. 


\section{References}

[1] V.E.Zakharov, Sov.Phys.JETP 60 (1971) 1012

[2] F.Gesztesy, W.Karwowski, Z.Zhao, Duke Math. Journ. 68 (1992) 101

[3] I.M.Krichever, unpublished

[4] P.D. Lax, Comm. Pure Appl. Math. 44 (1991) 1047

[5] G.A.El, A.L.Krylov, Stochastic Soliton Lattices, Report at the Intern. Conference 'Solitons, Geometry and Topology: on the Crossroads' in honor of the 60-th burthday of S.P. Novikov, Moscow (1998); solv-int/9810013.

[6] A.L.Krylov, G.A. El, Soviet Math. Surveys 54 (1999) 439.

[7] L.A.Pastur, A.L.Figotin, Spectra of Random and Almost-Periodic Operators, SpringerVerlag, New York (1992) .

[8] R.Johnson and J.Moser, Comm.Math.Phys. 84 (1982) 403.

[9] H.L. Frish, S.R. Lloyd, Phys. Rev. 120 (1960) 1179.

[10] I.M.Lifshitz, S.A.Gredeskul, A.L.Pastur, Introduction to the Theory of Disordered Systems, Wiley, New York (1982).

[11] B.A.Dubrovin and S.P.Novikov, Russian Math. Surveys 44 (1989) 35 .

[12] H. Flaschka, G. Forest, D.W. McLaughlin, Comm. Pure Appl. Math. 33 (1979) 739.

[13] S.P. Novikov, Func. Anal.Pril. 8 (1974) 54.

[14] P.D. Lax, Comm.Pure Appl. Math. 26 (1975) 141.

[15] Ito, Stochastic Processes, Mir, Moscow (1970).

[16] I.P.Cornfeld, S.V.Fomin, Ya.G.Sinai, Ergodic Theory Springer-Verlag, New York (1982).

[17] Yu.V. Prokhorov, Yu.A.Rozanov, Probability Theory, Nauka, Moscow (1970).

[18] M.I.Weinstein, J.B.Keller, SIAM Jornal Appl.Math. 47(1987) 941 .

[19] S. Venakides, AMS Transaction 301 (1987) 189.

[20] S. Venakides, Comm.Pure Appl.Math. 42 (1989) 711.

[21] P.D.Lax and C.D.Levermore, Comm. Pure Appl. Math. 36 (1983) 253,571,809.

[22] S.Kotani, private communication 
[23] V.A. Marchenko, Cauchy Problem for the KdV Equation with Nondecaying Initial Data, in: Integrability and Kinetic Equations for Solitons, eds. V.E. Zakharov et.al, Kiev (1990)

[24] Ya.G.Sinai, Introduction to ergodic theory, Phasis, Moscow (1996).

[25] M.Abramowitz and I.Stegun, Handbook of Mathematics Functions, Dover Publications, New York (1965) 